\title{
ATRIBUTOS FÍSICOS DE UM LATOSSOLO VERMELHO CULTIVADO COM PLANTAS DE COBERTURA, EM SEMEADURA DIRETA
}

\author{
José Luiz Rodrigues Torres ${ }^{(1) *}$, Marcos Gervasio Pereira ${ }^{(2)}$, Renato Lara de Assis ${ }^{(3)}$ e Zigomar
}

Menezes de Souza ${ }^{(4)}$

(1) Instituto Federal do Triângulo Mineiro, Campus Uberaba, Uberaba, Minas Gerais, Brasil.

(2) Universidade Federal Rural do Rio de Janeiro, Departamento de Solos, Seropédica, Rio de Janeiro, Brasil.

(3) Instituto Federal Goiano, Campus Iporá, Iporá, Goiás, Brasil.

(4) Universidade Estadual de Campinas, Faculdade de Engenharia Agrícola, Campinas, São Paulo, Brasil.

* Autor correspondente.

E-mail: jlrtorres@iftm.edu.br

\section{RESUMO}

A utilização do sistema de semeadura direta associado ao uso de plantas de cobertura e à rotação de culturas altera alguns atributos físicos do solo e pode indicar mudança em sua qualidade. Este estudo objetivou utilizar o índice de estabilidade de agregados (IEA) e o índice de sensibilidade (IS) para avaliar alterações nos atributos físicos do solo com o uso de diferentes plantas de cobertura após 12 anos de semeadura direta. Com delineamento de blocos ao acaso, utilizaram-se as coberturas: crotalária, milheto, sorgo, braquiária, pousio (vegetação espontânea), amostradas no ano de 2012, mais uma testemunha sem cobertura (semeadura convencional) desde o ano de 2000 , em que foram coletadas três amostras por parcela, perfazendo um total de 12 amostras por tratamento, com quatro repetições. Foram avaliadas a densidade do solo (Ds), macroporosidade (Ma), microporosidade (Mi), porosidade total (PT), estabilidade dos agregados (EA), diâmetro médio geométrico (DMG) e ponderado (DMP), índice de sensibilidade (Is) e índice da porcentagem de agregados com diâmetro superior a $2 \mathrm{~mm}$ (AGRI). A utilização das diferentes coberturas e a introdução do sistema de semeadura direta após 12 anos causaram alterações positivas nos atributos físicos na camada superficial do solo. As correlações positivas e negativas significativas entre densidade do solo e os outros atributos físicos avaliados evidenciaram a sua importância como bom indicador da qualidade do solo. Por meio do índice de sensibilidade, constatou-se que microporosidade 


\begin{abstract}
aumentou mais do que os outros atributos físicos após a implantação do sistema de semeadura direta na área.
\end{abstract}

Palavras-chave: plantio direto, agregação, densidade do solo.

\title{
ABSTRACT: PHYSICAL PROPERTIES OF AN OXISOL WITH COVER CROPS IN NO-TILLAGE
}

The use of no-tillage systems associated with the use of cover crops and crop rotation changes some soil physical properties and may indicate change in soil quality. This study aimed to use the aggregate stability index (IEA) and the sensitivity index (SI) to assess changes in soil physical attributes with the use of different plant cover after twelve years of no-tillage. We used a randomized block design with the following types of cover crops: sunn hemp, millet, sorghum, brachiaria, and fallow (natural vegetation) sampled in 2012, and a control (conventional planting) since 2000, in which three samples were collected per plot for a total of 12 samples per treatment, with four replications. We evaluated soil bulk density (Bd), macroporosity (Ma), microporosity (Mi), total pore volume (TPV), aggregate stability (AS), geometric mean diameter (GMD), mean weighted diameter (MWD), sensitivity index (SI), and index of the percentage of aggregates with diameter greater than $2 \mathrm{~mm}$ (AGRI). The use of different crop covers and the introduction of the no-tillage seeding system after twelve years caused positive changes in the physical properties of the soil surface layer. The positive and negative correlations between and significant soil density and the other evaluated physical attributes showed its importance as an indicator of soil quality. Through the sensitivity index, it was observed that microporosity increased more than the other physical attributes after the implementation of the no-tillage system in the area.

Keywords: no-tillage, aggregation, soil bulk density.

\section{INTRODUÇÃO}

No sistema de semeadura direta ocorre o acúmulo dos resíduos vegetais das culturas antecessoras na superfície do solo, causando o contínuo aporte de matéria orgânica na camada superficial e melhorias nos seus atributos físicos e químicos (Guareschi et al., 2012).

Alguns desses atributos físicos têm sido utilizados como indicadores de mudanças na qualidade do solo; contudo, esses devem ser sensíveis para refletir a influência dos sistemas de manejo e clima numa escala de tempo que permita a verificação de suas alterações (Islam e Weil, 2000). Os indicadores físicos que têm sido usados com frequência para aferir os impactos causados pelos sistemas de manejo são a densidade do solo (Ds), agregação, compactação, macroporosidade (Ma), microporosidade (Mi), porosidade total $(\mathrm{PT})$, capacidade de retenção d'água e estabilidade de agregados (EA) (Ramos et al., 2010; Pezarico et al., 2013).

Diversos estudos evidenciaram menores valores de Ds e maiores valores de PT em área nativa, quando comparada a outros sistemas de manejo (preparo convencional, pastagem e semeadura direta) (Demarchi et al., 2011; Torres et al., 2011; Guareschi et al., 2012). Essas alterações são atribuídas ao maior acúmulo de resíduos vegetais na superfície do solo e de C orgânico nas camadas superficiais e à menor alteração antrópica (Torres e Pereira, 2008). O maior aporte de resíduos na superfície do solo que ocorre no sistema de semeadura direta (SSD) causa o aumento do teor de $\mathrm{C}$ na camada superficial, que, associado às raízes das plantas de cobertura e das culturas comerciais após serem manejadas ou colhidas, proporciona a recuperação da agregação com maior volume total de poros no solo (Lima et al., 2013).

Em algumas áreas, após três anos de implantação do SSD, têm sido observado o aumento dos valores de Ds e Mi, com diminuição dos valores de Ma e PT na camada superficial, quando comparado ao sistema de semeadura convencional (SSC); contudo, a Ds tende aumentar com o aumento do teor de matéria orgânica no solo (Silva e Rosolem, 2001; Stone e Silveira, 2001; Hernani e Salton, 2009; Lago et al., 2012). O aumento da Ds e Mi devem-se ao efeito do tráfego de máquinas nas operações de plantio, manejo e colheita. Avaliando uma área sob SSD após 20 anos, Guareschi et al. (2012) observaram menores valores de Ds e maiores de PT, quando comparados a outras áreas com três e 15 anos sob o mesmo sistema. A justificativa é de que nos primeiros anos ocorre aumento na Ds pela reorganização das partículas de solo, que, após a consolidação do sistema, ocorre aumento nos teores de C orgânico, o que leva à redução na Ds e consequentemente 
ocorre o aumento da PT. Numa área após 23 anos de SSD, Hickmann et al. (2012) observaram que as alterações são significativas e positivas na camada superficial (0,00-0,05 m), quando comparado às áreas sob SSC. Nas profundidades maiores, os resultados evidenciaram que no SSC há aumento da degradação física do solo, que pode ser comprovado pelas reduções da Ma e PT, pela estabilidade de agregados, pela condutividade hidráulica, pelo carbono orgânico total e pelo aumento da Ds.

Alguns atributos físicos podem ser analisados por meio de índices. A compactação pode ser estimada pela resistência do solo à penetração (RP), enquanto o tamanho, a agregação e a estabilidade dos agregados do solo podem ser determinados por meio do diâmetro médio ponderado (DMP), diâmetro médio geométrico (DMG), índice de estabilidade dos agregados (IEA) (Bilibio et al., 2010) e índice da porcentagem de agregados com diâmetro superior a $2 \mathrm{~mm}$ (AGRI) (Wendling et al., 2005). Também se pode utilizar o índice de sensibilidade (Is) para avaliar a influência de diferentes tipos de cobertura vegetal sobre os atributos físicos do solo, pois esse índice determina a semelhança existente entre duas ou mais áreas e pode ser usado para determinar o grau de alteração entre os atributos das áreas estudadas (Bolinder et al., 1999; Pereira et al., 2010).

Esses indicadores e índices quando utilizados de forma conjunta podem indicar as alterações ocorridas nos sistemas de manejo do solo. Torres et al. (2011) aplicaram o Is para os atributos $\mathrm{Ds}$, Ma, Mi e PT, comparando SSC, SSD, pastagem (PAST) e campo nativo (CN), e observaram que as maiores variações do Is ocorreram para Ma nas camadas superficiais, pois de 0,00-0,05 m o menor valor ocorreu para SSC e o maior para PAST. Para 0,05-0,10 m, essa situação se inverteu, sendo o menor valor encontrado para PAST e o maior para SSC, enquanto para SSD o Is permaneceu constante. Fontana et al. (2010) compararam áreas cultivadas com cana-de-açúcar e pastagem sobre dois tipos de solos e notaram que os valores de Is nos solos sob pastagem foram maiores quando comparados aos da cana. Esses autores concluíram que as áreas cultivadas com pastagem estão mais próximas das condições naturais, sendo esse comportamento decorrente da melhor agregação do solo.

É comum a utilização de indicadores e índices, analisados de forma separada, para avaliar a qualidade física do solo em estudos de curta duração; entretanto, em estudos conduzidos por longos períodos e avaliados de forma conjunta ainda carecem ser melhor avaliados. Este estudo objetivou utilizar o índice de estabilidade de agregados (IEA) e o índice de sensibilidade (IS) para avaliar alterações nos atributos físicos do solo com o uso de diferentes plantas de cobertura após 12 anos de semeadura direta.

\section{MATERIAL E MÉTODOS}

O estudo foi conduzido na área experimental do Instituto Federal do Triângulo Mineiro (IFTM), localizado no município de Uberaba, MG, entre as coordenadas $19^{\circ} 39^{\prime} 19^{\prime \prime}$ de latitude sul e $47^{\circ} 57^{\prime} 27^{\prime \prime}$ de longitude oeste e altitude média de $795 \mathrm{~m}$. A área em estudo apresentava um histórico de mais de 20 anos com cultivo de milho e soja rotacionado em sistema de semeadura convencional (SSC) e estava há mais de um ano em pousio antes da instalação dos experimentos. Em 2000, fez-se o preparo do solo na área com subsolagem profunda $(0,45 \mathrm{~m})$, grade aradora e niveladora. $\mathrm{O}$ solo da área experimental foi caracterizado como Latossolo Vermelho distrófico (Embrapa, 2013), textura média, relevo local suave ondulado, apresentando na camada de 0,00-0,20 m: $210 \mathrm{~g} \mathrm{~kg}^{-1}$ de argila; $710 \mathrm{~g} \mathrm{~kg}^{-1}$ de areia; $80 \mathrm{~g} \mathrm{~kg}^{-1}$ de silte; $\mathrm{pH}\left(\mathrm{H}_{2} \mathrm{O}\right) 6,3 ; 19 \mathrm{mg} \mathrm{dm}^{-3}$ de $\mathrm{P}$ (Mehlich-1); $2,9 \mathrm{mmol}_{\mathrm{c}} \mathrm{dm}^{-3}$ de K; $22 \mathrm{mmol}_{\mathrm{c}} \mathrm{dm}^{-3} \mathrm{de} \mathrm{Ca}$; $10 \mathrm{mmol}_{\mathrm{c}} \mathrm{dm}^{-3}$ de $\mathrm{Mg} ; 20 \mathrm{mmol}_{\mathrm{c}} \mathrm{dm}^{-3} \mathrm{de} \mathrm{H}+\mathrm{Al}$; e $16 \mathrm{~g} \mathrm{dm}^{-3}$ de matéria orgânica e saturação por bases de $68 \%$.

O clima da região é classificado como Aw, tropical quente, segundo a classificação de Köppen, tendo verão quente e chuvoso e inverno frio e seco. $\mathrm{Na}$ região, ocorrem médias anuais de precipitação pluvial, temperatura e umidade relativa do ar de $1.600 \mathrm{~mm}, 22,6{ }^{\circ} \mathrm{C}$ e $68 \%$, respectivamente (Uberaba, 2009). Em 2012, a precipitação pluvial total foi de $1.780 \mathrm{~mm}$.

O delineamento utilizado foi de blocos ao acaso, com quatro repetições, em parcelas de 9,0 m de comprimento por $7,2 \mathrm{~m}$ de largura, perfazendo uma área total de $64,8 \mathrm{~m}^{2}$. Os tratamentos constaram das seguintes coberturas do solo: crotalária (Crotalaria juncea), milheto ADR 500 (Pennisetum glaucum L.), sorgo (Sorghum bicolor (L.) Moench), braquiária (Urochloa brizantha cv marandu), pousio (vegetação espontânea composta basicamente de Poáceas) amostradas em 2012, mais uma testemunha sem cobertura (semeadura convencional) desde o ano de 2000, em que foram coletadas três amostras por parcela, perfazendo o total de 12 amostras por tratamento.

Após as avaliações iniciais em 2000, na área foi cultivado o milheto comum; a partir de 2004, passou a ser utilizado o milheto ADR500. Entre o ano de 2000 e 2012, utilizaram-se as mesmas coberturas nas áreas, rotacionando o milho e a soja cultivados sobre os resíduos culturais dessas coberturas em relação ao ano anterior. Essas coberturas eram semeadas e avaliadas em duas épocas do ano, uma no período de abril a julho (período seco) e outra, de setembro a novembro (inicio do período chuvoso). Em 2003, toda área permaneceu em pousio até o plantio das culturas anuais (milho e soja). 
A sulcagem do solo e a semeadura das culturas de cobertura de solo foram realizadas de forma mecanizada. Utilizou-se espaçamento de $0,25 \mathrm{~m}$ entre as linhas de plantio e foram empregadas $25,60,25$ e 50 sementes por metro de crotalária, milheto, sorgo e braquiária, respectivamente. $\mathrm{Na}$ área de pousio, as plantas desenvolveram-se a partir de bancos de sementes existentes no local, onde se observou o predomínio de Poáceas.

Quando $50 \%$ das plantas de coberturas do solo atingiram o ponto de máximo de florescimento, foi realizada a dessecação dessas aplicando-se $1.440 \mathrm{~g} \mathrm{ha}^{-1}$ de Glifosato $+600 \mathrm{~g} \mathrm{ha}^{-1}$ de Paraquat. Quinze dias após o manejo das coberturas (dessecação), subdividiu-se as parcelas ao meio, que ficaram com áreas de 9,0 $\mathrm{m}$ de comprimento por 3,6 m de largura, perfazendo área total de $32,4 \mathrm{~m}^{2}$. A seguir, fez-se a semeadura de milho e soja nessas subparcelas sobre os resíduos vegetais das coberturas existentes sobre o solo, rotacionando o cultivo dessas culturas em relação ao ano anterior.

Em 2000, a amostragem para avaliação dos atributos físicos da área foi realizada antes do preparo do solo para implantação do sistema de semeadura direta. Em 2012, a amostragem foi realizada logo após a colheita do milho e da soja.

A densidade do solo (Ds) foi determinada em amostras com estrutura indeformada pelo método do anel volumétrico, que foram coletadas em anéis de $48 \mathrm{~mm}$ de diâmetro por $53 \mathrm{~mm}$ de altura, com o trado de Uhland, nas profundidades de 0,00-0,10; $0,10-0,20 ; 0,20-0,30 ;$ e $0,30-0,40 \mathrm{~m}$, sendo as amostras secas a $105^{\circ} \mathrm{C}$ por $24 \mathrm{~h}$. A distribuição de poros por tamanho foi determinada em amostras com estrutura indeformada, previamente saturadas por $24 \mathrm{~h}$, utilizando uma unidade de sucção a $0,60 \mathrm{~m}$ de altura de coluna de água. A macroporosidade (Ma), porosidade total (PT) e microporosidade (Mi) foram definidas segundo Embrapa (2011).

Foram coletadas três amostras deformadas em cada parcela, com auxílio de enxadão, nas profundidades de $0,00-0,10 ; 0,10-0,20$; e $0,20-0,30$ $\mathrm{m}$ para análise da estabilidade dos agregados (EA) por meio do método descrito por Kemper e Chepil (1965). A partir dos valores de massa dos agregados, foi calculado o diâmetro médio ponderado (DMP) (Equação 1), que é maior quanto maior for a porcentagem de agregados grandes retidos nas peneiras com malhas maiores; logo, a presença de agregado de maior tamanho ou maior DMP pode refletir a resistência do solo à erosão. $\mathrm{O}$ diâmetro médio geométrico (DMG) representa uma estimativa do tamanho da classe de agregados de maior ocorrência e pode ser estimado pela equação 2 .

$\mathrm{O}$ índice de estabilidade de agregados (IEA) (Equação 3) representa uma medida da agregação total do solo e não considera a distribuição por classe de agregados; logo, quanto maior a quantidade de agregado, $<0,25 \mathrm{~mm}$, menor será o IEA. O índice da porcentagem de agregados com diâmetro superior a $2 \mathrm{~mm}$ (AGRI) representa a proporção de agregados maiores que $2 \mathrm{~mm}$, (Equação 4). Esses índices de agregação do solo foram calculados da seguinte maneira:

$$
\mathrm{DMP}=\sum(\mathrm{xi} \times \text { wi }) \quad \text { Eq. } 1
$$

em que xi é o diâmetro médio das classes (mm); e wi, a proporção de cada classe em relação ao total (Wendling et al., 2005);

$$
\mathrm{DMG}=\exp \left\{\sum[\mathrm{ln}(\mathrm{xi}) \times \mathrm{PI}] / \sum \mathrm{PI}\right\} \quad \text { Eq. } 2
$$

em que $\ln$ [xi] é o logaritmo natural do diâmetro médio das classes; e PI, o peso (g) retido em cada peneira (Demarchi et al., 2011);

$$
\mathrm{IEA}=[(\mathrm{PA}-\mathrm{wp}<0,25) /(\mathrm{PA})] \times 100 \quad \text { Eq. } 3
$$

em que PA é o peso da amostra; wp $<0,25$ corresponde ao peso dos agregados da classe $<0,25 \mathrm{~mm}$, dado em g (Demarchi et al., 2011);

$$
\text { AGRI }=w i>2 \times 100 \quad \text { Eq. } 4
$$

em que wi $>2$ representa a proporção de agregados $>2 \mathrm{~mm}$, segundo Wendling et al. (2005).

Para comparar os valores de Ds, Ma, Mi e PT das áreas em 2000 e 2012, utilizou-se o índice de sensibilidade (Is) sugerido por Bolinder et al. (1999), que estima a intensidade das alterações dos atributos físicos do solo em razão das diferentes coberturas utilizadas. Esse índice foi calculado por meio da equação 5:

$$
\text { Is }=\text { as } / \text { ac }
$$

em que Is é o índice de sensibilidade; "as", o valor da variável (Ds, Ma, Mi e PT) da área considerada em cada cobertura no ano avaliado; e "ac", o valor da variável obtida na área sem cobertura (sistema de semeadura convencional) em 2000. Quanto mais próximo da unidade estiver o Is, menor será a alteração provocada nos atributos avaliados (Bolinder et al., 1999).

Os resultados encontrados foram analisados quanto à normalidade e homogeneidade dos dados por meio dos testes de Lilliefors, Cochran e Barttlet. Os atributos físicos e os índices avaliados foram submetidos à análise de correlação de Pearson e a análise de variância realizada com auxílio do programa estatístico SISVAR; nesses, foram aplicados o teste $\mathrm{F}$ para significância e as médias comparadas pelo teste de Tukey $(p<0,05)$.

\section{RESULTADOS E DISCUSSÃO}

Analisando os valores de Ds, Ma, Mi e PT em 2000, pode-se evidenciar a desestruturação do solo na profundidade até $0,30 \mathrm{~m}$, pois não ocorreram 
diferenças $(p<0,05)$ entre as camadas avaliadas (Quadro 1). Essa condição pode ser justificada pelo intenso preparo do solo que ocorreu nessa área por longo período, com duas a três gradagens antes do plantio, sempre na mesma profundidade, que, associado à textura média do solo, aumenta essa desestruturação após sucessivos cultivos.

Após a implantação do SSD com utilização de diferentes coberturas do solo, nos últimos 12 anos e nessa mesma área, foram analisadas as mesmas variáveis (Ds, Ma, Mi e PT). Observou-se que para Ds os valores diminuíram na camada de 0,10-0,20 m para crotalária, milheto, pousio e sorgo, que de forma geral a Ma diminuiu e Mi e PT aumentaram significativamente, quando comparados aos resultados de 2000 (Quadro 1). Apesar da diminuição da Ma, essa não atingiu o valor limite de $10 \%$, que representa o valor de referência que limita o crescimento de raízes, percolação de água e trocas gasosas (Tormena et al., 1998). Essas alterações ocorridas nesses atributos comprovam a reestruturação e estabilização das partículas desse solo. Mesmo assim, os valores obtidos para Ds ainda estão dentro do limite crítico para o desenvolvimento das culturas de $1,60 \mathrm{~kg} \mathrm{dm}^{-3}$, com exceção da camada de 0,10-0,20 m, estabelecido por Silva e Rosolem (2001). Esses valores de Ds evidenciam o efeito do rodado das máquinas agrícolas em todas as operações de cultivo na área. Segundo Lago et al. (2012), após três a quatro anos da implantação

Quadro 1. Valores de densidade do solo (Ds), macroporosidade (Ma), microporosidade (Mi), porosidade total (PT), diâmetro médio ponderado (DMP) e médio geométrico (DMG), índice de estabilidade de agregados (IEA) e da porcentagem de agregados com diâmetro superior a 2 mm (AGRI) observados em 2000 e após 12 anos de implantação do sistema de semeadura direta (SSD) na área, em 2012, em Latossolo Vermelho distrófico

\begin{tabular}{|c|c|c|c|c|c|c|c|c|}
\hline Profundidade & Ds & Ma & Mi & PT & DMP & DMG & IEA & AGRI \\
\hline \multirow[t]{3}{*}{$\mathrm{m}$} & $\mathrm{kg} \mathrm{dm}^{-3}$ & & $\%$ & & \multicolumn{2}{|c|}{$\mathrm{mm}$} & \multicolumn{2}{|c|}{$\%$} \\
\hline & \multicolumn{8}{|c|}{2000} \\
\hline & \multicolumn{8}{|c|}{ Sem cobertura vegetal } \\
\hline $0,00-0,10$ & $1,62 \mathrm{Aa}^{\mathrm{ns}}$ & $29,73 \mathrm{Aa}^{*}$ & $12,60 \mathrm{Bb}$ & $38,88 \mathrm{Ba}$ & $3,29 \mathrm{Da}^{*}$ & $1,74 \mathrm{Ea}^{*}$ & $78,5 \mathrm{Ca}$ & $28,9 \mathrm{Da}$ \\
\hline $0,10-0,20$ & $1,66 \mathrm{Aa}$ & $24,43 \mathrm{Ab}$ & $16,71 \mathrm{Ba}$ & $37,30 \mathrm{Ba}$ & $2,75 \mathrm{Bb}$ & $1,31 \mathrm{Bb}$ & $75,5 \mathrm{Db}$ & $22,6 \mathrm{Cb}$ \\
\hline \multirow[t]{3}{*}{$0,20-0,30$} & $1,58 \mathrm{Aa}$ & $24,91 \mathrm{Ab}$ & $17,95 \mathrm{Ba}$ & $40,41 \mathrm{Ba}$ & $2,10 \mathrm{Cc}$ & $0,90 \mathrm{Cc}$ & $71,3 \mathrm{Cc}$ & $16,0 \mathrm{Dc}$ \\
\hline & \multicolumn{8}{|c|}{2012} \\
\hline & \multicolumn{8}{|c|}{ Braquiária } \\
\hline $0,00-0,10$ & 1,59 Aab & $14,70 \mathrm{Ba}$ & $28,80 \mathrm{Aa}$ & $45,28 \mathrm{Aab}$ & $3,92 \mathrm{Ba}$ & $2,55 \mathrm{Bb}$ & $90,6 \mathrm{Ba}$ & $38,1 \mathrm{Ba}$ \\
\hline $0,10-0,20$ & $1,66 \mathrm{Aa}$ & $14,76 \mathrm{Ba}$ & $27,88 \mathrm{Aa}$ & $43,03 \mathrm{Ab}$ & $3,05 \mathrm{Ab}$ & $1,69 \mathrm{Aa}$ & $88,5 \mathrm{Bb}$ & $27,5 \mathrm{Ab}$ \\
\hline \multirow[t]{2}{*}{$0,20-0,30$} & $1,49 \mathrm{Ab}$ & $18,45 \mathrm{Ba}$ & $27,80 \mathrm{Aa}$ & $48,86 \mathrm{Aa}$ & $3,02 \mathrm{Ab}$ & $1,67 \mathrm{Aa}$ & $88,5 \mathrm{Ab}$ & $27,2 \mathrm{Ab}$ \\
\hline & \multicolumn{8}{|c|}{ Crotalária } \\
\hline $0,00-0,10$ & $1,58 \mathrm{Aa}$ & $16,90 \mathrm{Ba}$ & $29,40 \mathrm{Aa}$ & $45,90 \mathrm{Aa}$ & $4,33 \mathrm{Aa}$ & $3,24 \mathrm{Aa}$ & $93,1 \mathrm{Aa}$ & $42,7 \mathrm{Aa}$ \\
\hline $0,10-0,20$ & $1,53 \mathrm{Ba}$ & $18,25 \mathrm{Ba}$ & $26,58 \mathrm{Aa}$ & $47,34 \mathrm{Aa}$ & $2,86 \mathrm{Bb}$ & $1,57 \mathrm{Ab}$ & $89,3 \mathrm{Ab}$ & $24,9 \mathrm{Bb}$ \\
\hline \multirow[t]{2}{*}{$0,20-0,30$} & $1,53 \mathrm{Aa}$ & $18,42 \mathrm{Ba}$ & $27,70 \mathrm{Aa}$ & $47,35 \mathrm{Aa}$ & $2,73 \mathrm{Bc}$ & $1,50 \mathrm{Bb}$ & $87,7 \mathrm{Ac}$ & $23,0 \mathrm{Cc}$ \\
\hline & \multicolumn{8}{|c|}{ Milheto } \\
\hline $0,00-0,10$ & 1,58 Aa & $16,04 \mathrm{Ba}$ & $31,17 \mathrm{Aa}$ & $45,89 \mathrm{Aa}$ & $3,23 \mathrm{Da}$ & $1,91 \mathrm{Da}$ & $89,4 \mathrm{Ba}$ & $39,6 \mathrm{Ba}$ \\
\hline $0,10-0,20$ & $1,46 \mathrm{Ca}$ & $14,92 \mathrm{Ba}$ & $27,26 \mathrm{Aa}$ & 49,93 $\mathrm{Aa}$ & $2,05 \mathrm{Db}$ & $1,08 \mathrm{Cb}$ & $87,5 \mathrm{Bb}$ & $15,4 \mathrm{Db}$ \\
\hline \multirow[t]{2}{*}{$0,20-0,30$} & $1,59 \mathrm{Aa}$ & $15,11 \mathrm{Ba}$ & $28,26 \mathrm{Aa}$ & $45,39 \mathrm{Aa}$ & $1,76 \mathrm{Dc}$ & 0,89 Cc & $85,0 \mathrm{Ac}$ & $12,2 \mathrm{Ec}$ \\
\hline & \multicolumn{8}{|c|}{ Pousio } \\
\hline $0,00-0,10$ & $1,55 \mathrm{Aa}$ & $15,89 \mathrm{Ba}$ & $39,25 \mathrm{Aa}$ & $46,74 \mathrm{Aa}$ & $3,61 \mathrm{Ca}$ & $2,20 \mathrm{Ca}$ & $90,4 \mathrm{Ba}$ & $34,4 \mathrm{Ca}$ \\
\hline $0,10-0,20$ & $1,57 \mathrm{Ba}$ & $16,06 \mathrm{Ba}$ & $27,70 \mathrm{Ab}$ & $46,15 \mathrm{Aa}$ & $1,82 \mathrm{Ec}$ & 0,81 Dc & $77,6 \mathrm{Cc}$ & $13,9 \mathrm{Db}$ \\
\hline \multirow[t]{2}{*}{$0,20-0,30$} & $1,61 \mathrm{Aa}$ & $16,40 \mathrm{Ba}$ & $28,95 \mathrm{Ab}$ & $44,67 \mathrm{Aa}$ & $2,04 \mathrm{Cb}$ & $0,96 \mathrm{Cb}$ & $83,8 \mathrm{Bb}$ & $16,0 \mathrm{Dc}$ \\
\hline & \multicolumn{8}{|c|}{ Sorgo } \\
\hline $0,00-0,10$ & 1,62 Aa & $13,94 \mathrm{Ba}$ & $30,94 \mathrm{Aa}$ & $44,46 \mathrm{Aa}$ & $4,34 \mathrm{Aa}$ & $3,20 \mathrm{Aa}$ & $92,3 \mathrm{Aa}$ & $42,9 \mathrm{Aa}$ \\
\hline $0,10-0,20$ & $1,57 \mathrm{Ba}$ & $17,20 \mathrm{Ba}$ & $27,22 \mathrm{Aa}$ & $46,12 \mathrm{Aa}$ & $2,49 \mathrm{Cc}$ & $1,19 \mathrm{Cc}$ & $84,5 \mathrm{Bb}$ & $21,1 \mathrm{Cc}$ \\
\hline $0,20-0,30$ & $1,61 \mathrm{Aa}$ & $16,08 \mathrm{Ba}$ & $28,17 \mathrm{Aa}$ & $44,70 \mathrm{Aa}$ & 2,83 $\mathrm{Bb}$ & $1,47 \mathrm{Bb}$ & $86,1 \mathrm{Ab}$ & $25,1 \mathrm{Bb}$ \\
\hline CV (\%) & 4,94 & 15,15 & 17,64 & 5,92 & 2,07 & 3,96 & 5,01 & 4,03 \\
\hline
\end{tabular}

${ }^{\mathrm{ns}}$ : não significativo; * significativo $(\mathrm{p}<0,05)$. Médias seguidas de mesmas letras maiúsculas na coluna comparam coberturas do solo nas mesmas profundidades, assim como as minúsculas na coluna comparam as profundidades dentro de cada cobertura avaliada, que não diferem entre si pelo teste de Tukey $(p<0,05)$. 
do SSD, é comum os solos apresentarem maiores valores de Ds e Mi na camada superficial e menores valores de Ma e PT, quando comparados com os solos em SSC. Esses autores afirmaram que isso ocorre em razão do arranjamento natural do solo não mobilizado e da pressão provocada pelo trânsito de máquinas e implementos agrícolas mais pesados.

Alguns outros estudos evidenciaram que as alterações nos atributos físicos ocorrem principalmente na camada superficial. Bertol et al. (2004) observaram que a Ds e Mi foram maiores e Ma e PT menores na área SSD na profundidade de 0,00-0,10 m, quando comparadas ao plantio convencional e campo nativo. Stone e Silveira (2001) observaram que após três anos ocorreram maiores valores de Ds e Mi e menores valores de Ma e PT na camada superficial em áreas em SSD, quando comparados a outros sistemas de manejo. Esses autores justificam que isso ocorre em razão do arranjamento natural das partículas do solo, que não foi revolvido, tendo menor transito de máquinas e implementos agrícolas, além do aumento do teor de matéria orgânica na camada superficial.

$\mathrm{Na}$ camada de 0,10-0,20 m, observou-se que ocorreram alterações $(p<0,05)$ para a Ds, que diminuiu nas áreas de crotalária, milheto, pousio e sorgo, quando comparadas à área sem cobertura (Quadro 1). Essas alterações podem ser justificadas pela elevada produção de biomassa dessas culturas, conforme já registrado em outros estudos na mesma área (Torres e Pereira, 2008). Além disso, o desenvolvimento radicular dessas plantas nos primeiros $0,20 \mathrm{~m}$, principalmente das Poáceas que é fasciculado, pode apresentar efeito rizosférico intenso e ao ser decomposto libera nutrientes e contribui para a formação da matéria orgânica do solo, favorecendo intensamente seu estado de agregação, conforme destacado por Torres e Pereira (2008).

Efeito semelhante foi observado por Hickmann et al. (2012), os quais relataram maiores valores de Ds na profundidade de 0,05-0,10 m, após 23 anos de semeadura direta. $\mathrm{O}$ aumento da Ds interfere no crescimento radicular, na redução da aeração, no aumento na RP e provoca alterações na dinâmica da água no solo. Hernani e Salton (2009) avaliaram os atributos do solo em diferentes sistemas de manejo por 16 anos e observaram que no SSD ocorreram a elevação da Ds e diminuição da PT nas profundidades de 0,00-0,05 e 0,05-0,10 $\mathrm{m}$ e o aumento dos agregados de maior tamanho e consequentemente do DMP. Esses autores destacaram ainda que a combinação entre o SSD e a rotação de culturas melhorou a fertilidade do solo, resultando em produtividade mais estável e elevada das culturas de soja e milho.

Após a determinação da estabilidade dos agregados, aplicaram-se os índices DMP, DMG, IEA e AGRI sobre os valores observados para o ano de 2000. Constatou-se que esses índices foram significativamente $(p<0,05)$ superiores na camada superficial $(0,00-0,10 \mathrm{~m})$ e diminuíram em profundidade (Quadro 1). Após 12 anos de implantação do SSD na área, com utilização de diferentes coberturas do solo para produção de biomassa, foram analisados os mesmos atributos e observou-se que todos os valores apresentaram padrão semelhante ao observado em 2000, sendo significativamente maiores na superfície e diminuindo em profundidade.

Por meio das variações ocorridas nesses índices, pode-se evidenciar que ocorreu a reestruturação do solo e a estabilização do sistema após esse período, o que se deve à ação das raízes e ao aporte de material orgânico ao longo do período avaliado, conforme também observado por Pereira et al. (2010), os quais afirmaram que quanto maior o valor de DMP e DMG, maior a contribuição do manejo na estabilização dos agregados do solo. Resultados semelhantes foram constatados por Hernani e Salton (2009), em estudos conduzidos por longos períodos.

Todos os IEA calculados foram significativamente maiores em 2012 , que variou entre 89,4 e $93,1 \%$; 89,3 e $77,6 \%$; e 87,7 e $83,8 \%$, quando comparados aos valores de 78,5; 75,5; e 71,3\% obtidos em 2000 , para as profundidades de $0,00-0,10 ; 0,10-0,20$; e 0,20-0,30 m, respectivamente (Quadro 1). Esses elevados valores de IEA são decorrentes da proteção proporcionada pelos resíduos vegetais das diferentes coberturas do solo, que protegem o solo contra a desagregação causada pelo impacto das chuvas e variações bruscas de umidade e fornecem energia da matéria orgânica para a atividade microbiana, que produz substâncias responsáveis pela formação e estabilização dos agregados, além do efeito agregador das raízes dessas plantas, fato esse destacado por Wendling et al. (2005) em estudo avaliando cinco sistemas de uso do solo e analisando os mesmos índices de agregação. Bertol et al. (2004) destacaram que o C orgânico é um dos principais fatores de formação e estabilização dos agregados, que, na ausência de preparo, os ciclos de umedecimento e secagem do solo são potencializados e consolidam a agregação.

Com relação ao índice AGRI, nas áreas com cobertura de milheto e pousio, nas profundidades de 0,10-0,20 e 0,20-0,30 m, os valores em 2012 foram menores quando comparados aos de 2000 , mesmo apresentando IEA variando entre 77,6 e $87,5 \%$, que comprova que houve maior distribuição dos agregados entre as peneiras, que pode ser justificado por maior desenvolvimento do sistema radicular fasciculado dessas plantas, que estão mais bem adaptadas às condições edafoclimaticas no local do estudo, conforme comprovado por Torres e Pereira (2008). Além disso, outros estudos conduzidos na mesma área experimental comprovam que essas coberturas estão entre aquelas que mais produzem fitomassa, deixando maior quantidade de resíduos sobre a superfície (Torres et al., 2008, 
2014), que, somados às raízes, influem nos teores de $\mathrm{C}$ orgânico do solo e influenciam positivamente a sua agregação.

Em estudo semelhante, Demarchi et al. (2011) compararam cinco sistemas de manejo, avaliaram DMP e DMG e IEA e AGRI e observaram os melhores índices na área de pastagem (Urochloa brizantha), que apresentaram os valores de 4,12 e $2,96 \mathrm{~mm}$; e 92,9 e 79,7\%, para pastagem; e 3,43 e 2,04 mm; e 88,4 e 63,7 \%, para mata nativa, respectivamente. Esses estudiosos justificaram que essas áreas apresentaram índices de estabilidade de agregados elevados em razão da maior agregação do solo promovida pelas raízes das plantas de cultivadas na área.

$O$ índice de sensibilidade (Is) foi calculado para os atributos Ds, Ma, Mi e PT para as áreas com coberturas em 2012, comparando-as à área sem cobertura de 2000 (Figura 1).

Analisando o índice de sensibilidade (Is) para os valores de Ds, observou-se que não ocorreram variações significativas $(p<0,05)$ entre as coberturas e profundidades avaliadas, quando comparadas às de 2000 e 2012, que se mantiveram constantes e próximas da unidade (Figura 1). A manutenção dos valores de Ds nessas camadas comprova que ocorreu a consolidação do SSD, em razão do não revolvimento do solo e aporte de material orgânico via resíduos vegetais na superfície e sistema radicular das plantas cultivadas na área; entretanto, era esperado que esses valores diminuíssem, uma vez que, como destacado por Guareschi et al. (2012), o aumento da matéria orgânica do solo decorrente dos sucessivos aportes de resíduos vegetais e raízes, após alguns anos de adoção do SSD, pode reduzir os valores de Ds na camada superficial. Contudo, isso não ocorreu de forma significativa. Possivelmente, esses valores elevados de Ds se devem ao efeito do tráfego em condições inadequadas de umidade, promovendo assim deformação do solo, resultando em aumento da Ds.

Com relação ao Is para $\mathrm{Ma}$, observou-se que os valores diminuíram nas áreas com cobertura (braquiária, crotalaria, milheto, pousio e sorgo), que foram significativamente menores que as áreas sem cobertura, além de ocorrer diferenças entre as profundidades avaliadas dentro de cada tratamento (Figura 1). Em áreas sob SSC, ocorre a redução dos agregados na camada mobilizada, que acelera a decomposição da matéria orgânica e reflete negativamente na resistência dos agregados; no entanto, quando comparado ao SSD, observa-se que no SSC tem ocorrido aumento da macroporosidade, permeabilidade e armazenamento de ar, facilitando o crescimento das raízes das plantas dentro da camada preparada (Hickmann et al., 2012).
Com relação ao Is para $\mathrm{Mi}$, os valores na profundidade de 0,00-0,10 m variaram de 1,5 a 2,3 para braquiária, crotalária e milheto; de 1,6 a 2,5 para o sorgo; e de 1,6 a 3,1 para o pousio em 2012, ou seja, aumentaram significativamente quando comparados aos do ano de 2000, principalmente na camada de 0,00-0,10 m (Figura 1). Esses resultados comprovaram que as maiores alterações ocorreram nas camadas superficiais $(0,00-0,10 \mathrm{~m})$ e que a reestruturação do solo ocorre de forma acelerada e significativa após a implantação do SSD. Ao comparar Latossolo e Argissolo cultivados com duas coberturas vegetais (cana-de-açúcar e pastagem), Fontana et al. (2010) observaram que os valores de Is dos solos sob pasto foram maiores que os sob cana. Os autores justificaram que os menores valores de Is encontrados na área de cana refletem o efeito prejudicial do sistema de preparo convencional utilizados na cultura sobre a agregação do solo.

Na mesma região, Torres et al. (2011) calcularam o Is para os atributos Ds, PT, Ma e Mi para os sistemas de manejo - plantio convencional (PC), pastagem (PAST), semeadura direta (SD) e Cerrado nativo $(\mathrm{CN})$-, em diferentes profundidades. Esses autores observaram que as maiores variações dos Is ocorreram para Ma no PC e PAST, se mantendo constante na área sob SD, enquanto para Ds, PT e Mi os valores ficaram próximos da unidade em todos os tratamentos.

Ao analisar as correlações existentes entre os indicadores e índices utilizados, observou-se que a Ds foi o atributo que se correlacionou diretamente com todos os outros, positivamente com Mi, DMP, DMG e AGRI e negativamente com Ma, PT e IEA, em 2000 e 2012, respectivamente (Quadro 2).

Essas correlações evidenciaram que à medida que a Ds aumentou, simultaneamente ocorreu a elevação da Mi, DMP, DMG e AGRI, evidenciando que a alteração do sistema de manejo está causando modificações nos atributos físicos do solo, principalmente na sua agregação, conforme comprovado pelos elevados IEA nas profundidades avaliadas (Quadro 1), concordando com os resultados de Lima et al. (2013), enquanto a correlação negativa $(p<0,05)$ entre a Ds com PT e IEA comprova a diminuição da capacidade de aeração do solo em profundidade, influenciando a infiltração da água, conforme observado por Hickmann et al. (2012) em estudo semelhante, numa área avaliada 23 anos após a implantação do SSD, onde observaram a mesma correlação negativa. Wendling et al. (2005), ao pesquisarem Latossolos submetidos a diferentes manejos, verificaram correlações positivas entre índices DMP, DMG, AGRI e IEA.

Segundo Pezarico et al. (2013), os solos com maior Ds apresentam diminuição da PT, redução da permeabilidade e da infiltração de água, destruição dos agregados e aumento da resistência mecânica à penetração, ocasionando prejuízo ou 


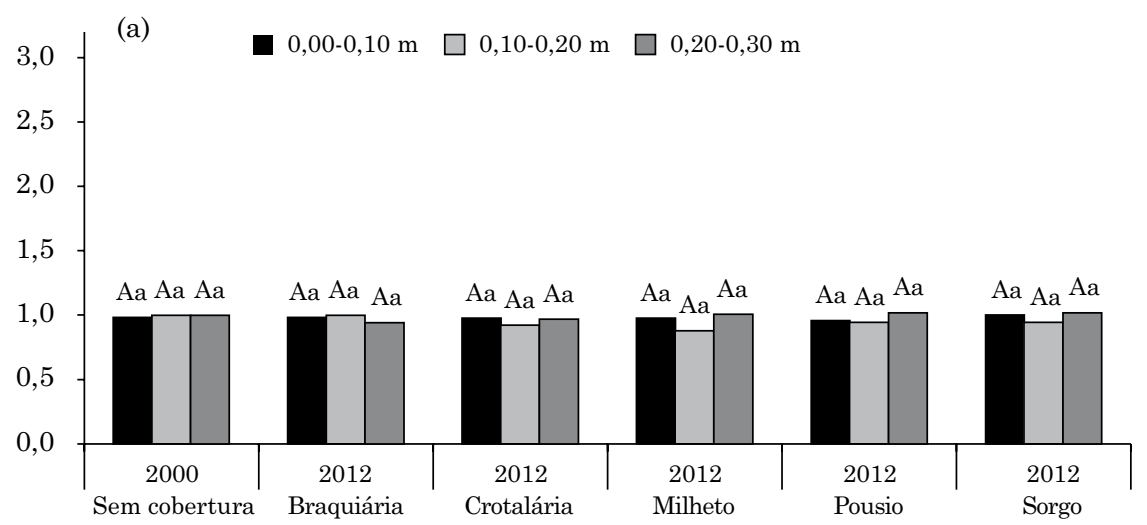

(b)

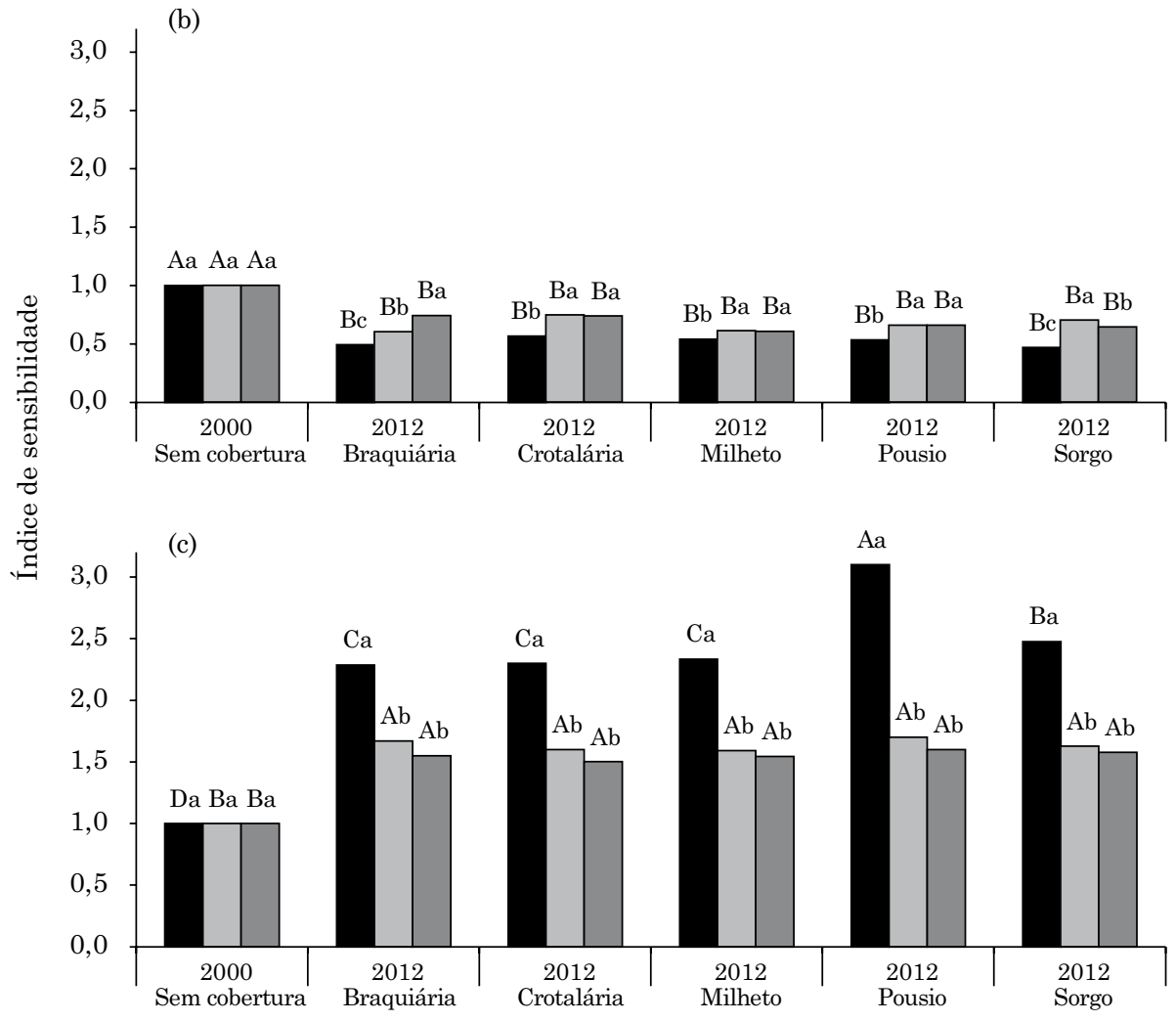

(d)

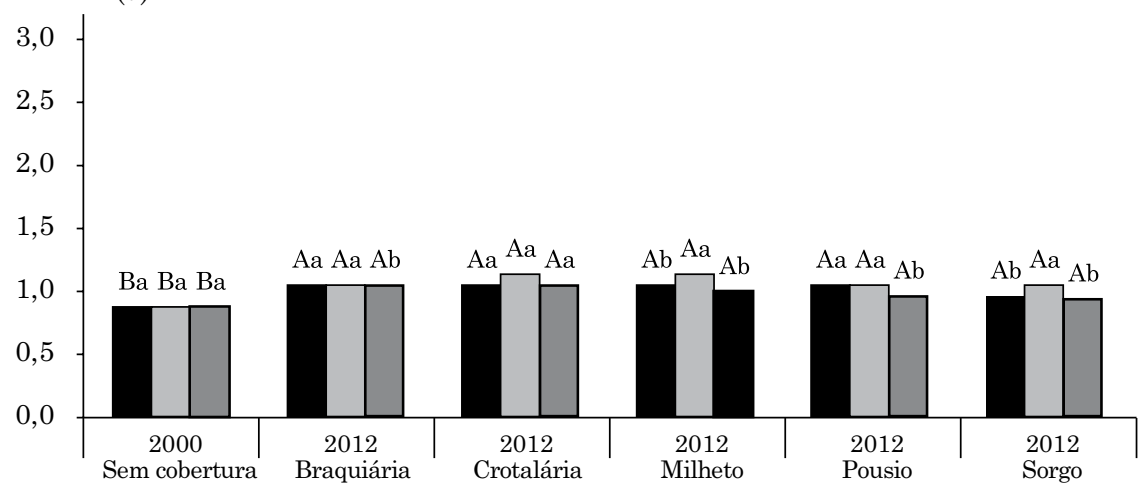

Figura 1. Índice de sensibilidade (Is) para densidade do solo (a), macroporosidade (b), microporosidade (c) e porosidade total (d), em Latossolo Vermelho distrófico. Médias seguidas de mesmas letras maiúsculas comparam coberturas nas mesmas profundidades, assim como as minúsculas comparam profundidades dentro das coberturas avaliadas e não diferem entre si pelo teste de Tukey $(p<0,05)$. 
Quadro 2. Coeficientes de correlações de Pearson entre indicadores e índices dos atributos físicos em 2000 e 2012, em Latossolo Vermelho distrófico

\begin{tabular}{|c|c|c|c|c|c|c|c|c|}
\hline \multirow[t]{2}{*}{ Variável } & \multicolumn{8}{|c|}{2000} \\
\hline & Ma & Mi & PT & DMP & DMG & IEA & AGRI & Is \\
\hline $\mathrm{Ds}$ & $-0,08^{*}$ & $0,22^{*}$ & $-0,99^{*}$ & $0,55^{*}$ & $0,49 *$ & $-0,58^{*}$ & $0,51^{*}$ & - \\
\hline $\mathrm{Ma}$ & & $-0,95^{* *}$ & $0,09^{\mathrm{ns}}$ & $0,79^{\text {ns }}$ & $0,83^{* *}$ & $0,77^{\mathrm{ns}}$ & $0,81^{\mathrm{ns}}$ & - \\
\hline $\mathrm{Mi}$ & & & $0,21^{\mathrm{ns}}$ & $-0,94^{\mathrm{ns}}$ & $-0,96^{* *}$ & $-0,92^{\mathrm{ns}}$ & $-0,95^{\mathrm{ns}}$ & - \\
\hline $\mathrm{PT}$ & & & & $-0,54^{\mathrm{ns}}$ & $-0,48^{\mathrm{ns}}$ & $-0,57^{\mathrm{ns}}$ & $-0,50^{\mathrm{ns}}$ & - \\
\hline DMP & & & & & $0,99^{\mathrm{ns}}$ & $0,99^{* *}$ & $0,99 *$ & - \\
\hline DMG & & & & & & $0,99^{*}$ & $0,99^{* *}$ & - \\
\hline \multirow[t]{3}{*}{ IEA } & & & & & & & $0,99^{\mathrm{ns}}$ & - \\
\hline & \multicolumn{8}{|c|}{2012} \\
\hline & $\mathrm{Ma}$ & $\mathrm{Mi}$ & $\mathrm{PT}$ & DMP & DMG & IEA & AGRI & Is \\
\hline Ds & $-0,48^{*}$ & $0,09^{*}$ & $-0,99^{*}$ & $0,23^{*}$ & $0,23^{*}$ & $-0,03^{*}$ & $0,02^{*}$ & $0,35^{*}$ \\
\hline $\mathrm{Ma}$ & & $-0,24^{*}$ & $0,47^{\mathrm{ns}}$ & $-0,15^{* *}$ & $-0,22^{* *}$ & $0,25^{*}$ & $0,39^{*}$ & $0,25^{*}$ \\
\hline $\mathrm{Mi}$ & & & $-0,09 * *$ & $0,43^{*}$ & $0,40^{*}$ & $-0,23^{*}$ & $-0,25^{*}$ & $-0,27 *$ \\
\hline $\mathrm{PT}$ & & & & $-0,23^{*}$ & $-0,22^{*}$ & $0,03^{*}$ & $-0,04^{*}$ & $-0,37^{*}$ \\
\hline DMP & & & & & $0,98^{\mathrm{ns}}$ & $0,32^{*}$ & $0,26^{*}$ & $-0,05^{*}$ \\
\hline DMG & & & & & & $0,25^{*}$ & $0,17^{*}$ & $-0,12^{*}$ \\
\hline IEA & & & & & & & $0,82^{*}$ & $0,66^{*}$ \\
\hline AGRI & & & & & & & & $0,63^{*}$ \\
\hline
\end{tabular}

Ds: densidade do solo; Ma: macroporosidade; Mi: microporosidade; PT: volume total de poros; DMP: diâmetro médio ponderado; DMG: diâmetro médio geométrico; IEA: índice de estabilidade dos agregados; AGRI: índice da porcentagem de agregados com diâmetro $>2 \mathrm{~mm}$; e Is: índice de sensibilidade. ${ }^{\mathrm{ns}}$ : não significativo; ${ }^{*} \mathrm{e}^{* *}$ : significativo $\mathrm{p}<0,01 \mathrm{e} \mathrm{p}<0,05$ pelo teste Tukey, respectivamente.

comprometendo a qualidade física do solo. Ramos et al. (2010) destacaram que a Ds é um dos atributos mais importantes e utilizados como indicador de qualidade do solo, pois é um componente sensível às alterações causadas pelo manejo. Beutler et al. (2004) observaram correlações da Ds com PT e Ma (negativas) e positivas com $\mathrm{Mi}$, justificando que isso ocorre em razão da aproximação das partículas com o incremento da pressão mecânica exercida sobre o solo, reduzindo a proporção dos poros de maior diâmetro e incrementando ligeiramente os de menor diâmetro.

\section{CONCLUSÕES}

A utilização das diferentes coberturas e a introdução do sistema de semeadura direta após 12 anos causaram alterações positivas nos atributos físicos na camada superficial do solo.

As correlações positivas e negativas significativas entre densidade do solo e os outros atributos físicos avaliados evidenciaram a sua importância como bom indicador da qualidade do solo.

Por meio do índice de sensibilidade, constatouse que a microporosidade aumentou mais do que os outros atributos físicos após a implantação do sistema de semeadura direta na área.

\section{AGRADECIMENTOS}

Ao Instituto Federal do Triângulo Mineiro, Campus Uberaba, pela infraestrutura disponibilizada; à Fundação de Amparo à Pesquisa do Estado de Minas Gerais (Fapemig); e ao Conselho Nacional de Desenvolvimento Científico e Tecnológico (CNPq), pelo financiamento do projeto e pela concessão da bolsa de estudo do primeiro autor e bolsas de produtividade em pesquisa dos demais autores.

\section{REFERÊNCIAS}

Bertol I, Albuquerque JA, Leite D, Amara, AJ, Zoldan Junior WA. Propriedades físicas do solo sob preparo convencional e semeadura direta em rotação e sucessão de culturas, comparadas às do campo nativo. $\mathrm{R}$ Bras Ci Solo. 2004;28:155-65.

Beutler AN, Centurion JF, Roque CG. Relação entre alguns atributos físicos e a produção de grãos de soja e arroz de sequeiro em Latossolos. Ci Rural. 2004;34:365-71.

Bilibio WD, Corrêa GF, Borges EN. Atributos físicos e químicos de um Latossolo, sob diferentes sistemas de cultivo. Ci Agrotec. 2010;34:817-22.

Bolinder MA, Angers DA, Gregorich EG, Carter MR. The response of soil quality indicators to conservation manage. Can J Soil Sci. 1999; 79:37-45. 
Demarchi JC, Perusi MC, Piroli ED. Análise da estabilidade de agregados de solos da microbacia do Ribeirão São Domingos, Santa Cruz do Rio Pardo - SP, sob diferentes tipos de uso e ocupação. R Bras Tecnol Aplic Ci Agric. 2011;4:7-29.

Empresa Brasileira de Pesquisa Agropecuária - Embrapa. Manual de métodos de análise de solo. $2^{\mathrm{a}}$ ed. Rio de Janeiro: Centro Nacional de Pesquisa de Solos; 2011.

Empresa Brasileira de Pesquisa Agropecuária - Embrapa. Sistema brasileiro de classificação de solos. $3^{\text {a }}$ ed. Brasília, DF; 2013.

Fontana A, Brito RJ, Pereira MG, Loss A. Índices de agregação e a relação com as substâncias húmicas em Latossolos e Argissolo de tabuleiros costeiros, Campos dos Goytacazes, RJ. R Bras Ci Agron. 2010:5:291-7.

Guareschi RF, Pereira MG, Perin A. Deposição de resíduos vegetais, matéria orgânica leve, estoques de carbono e nitrogênio e fósforo remanescente sob diferentes sistemas de manejo no Cerrado goiano. R Bras Ci Solo. 2012;36:909-20.

Hernani LC, Salton JC. Atributos de solo e produtividade de culturas em sistemas de manejo conduzidos por dezesseis anos. Bol Pesq Desenv. 2009;54:1-63.

Hickmann C, Costa LM, Schaefer CEG, Fernandes RBA, Andrade CLT. Atributos físico-hídricos e carbono orgânico de um Argissolo após 23 anos de diferentes manejos. Caatinga. 2012;25:128-36.

Islam KR, Weil RR. Soil quality indicator properties in midAtlantic soils as influenced by conservation management. J Soil Water Conserv. 2000;55:69-79.

Kemper WD, Chepil WS. Size distribution of aggregation. In: Black CA, editor. Methods of soil analysis. Madison: America Society of Agronomy; 1965. p.499-510. (Agronomy monoghaph, 9).

Lago WNM, Lacerda MPC, Neumann MRB. Indicadores de qualidade dos solos na microbacia do Ribeirão Extrema, DF: Parte II. R Bras Eng Agric Amb. 2012;16:721-9.

Lima JSS, Silva SA, Silva JM. Variabilidade espacial de atributos químicos de um Latossolo Vermelho-Amarelo cultivado em plantio direto. R Ci Agron. 2013;44:16-23.

Pezarico CR, Vitorino ACT, Mercante FM, Daniel O. Indicadores de qualidade do solo em sistemas agroflorestais. R Ci Agron. 2013;56:40-7.
Ramos FT, Monari YC, Nunes MCM, Campos DTS, Ramos DT. Indicadores de qualidade em um Latossolo Vermelho-Amarelo sob pastagem extensiva no pantanal matogrossense. Caatinga. 2010;23:112-20.

Pereira MG, Loss A, Beutler SJ, Torres JLR. Carbono, matéria orgânica leve e P remanescente em diferentes sistemas de manejo do solo. Pesq Agropec Bras. 2010;45:508-14.

Silva RH, Rosolem CA. Crescimento radicular de espécies utilizadas como cobertura decorrente da compactação do solo. $\mathrm{R}$ Bras Ci Solo. 2001;25:253-60.

Stone LF, Silveira PM. Efeitos do sistema de preparo e da rotação de culturas na porosidade e densidade do solo. R Bras Ci Solo. 2001;25:395-401.

Tormena CA, Silva AP, Libardi PL. Caracterização do intervalo hídrico ótimo de um Latossolo Roxo sob plantio direto. R Bras Ci Solo. 1998;22:573-81.

Torres JLR, Pereira MG. Dinâmica do potássio nos resíduos vegetais de plantas de cobertura no Cerrado. R Bras Ci Solo. 2008;32:1609-18.

Torres JLR, Pereira MG, Fabian AJ. Produção de fitomassa por plantas de cobertura e mineralização de seus resíduos em plantio direto. Pesq Agropec Bras. 2008;43:421-8.

Torres JLR, Fabian AJ, Pereira MG. Alterações dos atributos físicos de um Latossolo Vermelho submetido a diferentes sistemas de manejo. Ci Agron. 2011;35:437-45.

Torres JLR, Pereira MG, Cunha MA, Vieira DMS, Rodrigues ES. Produtividade do milho cultivado em sucessão a crotalária, milheto e braquiária no cerrado mineiro. Enciclop. Biosfera. 2014;18:2482-91.

Uberaba. [Brasil] Prefeitura Municipal. Uberaba em dados. Edição 2009 [acesso em 21 nov 2011]. Disponível em: http:// www.uberaba.mg.gov.br/portal/acervo/desenvolvimento/arquivos/ uberaba_em_dados/Edicao_2009/Capitulo01.pdf.

Wendling B, Jucksch I, Mendonça ES, Neves JCL. Carbono orgânico e estabilidade de agregados de um Latossolo Vermelho sob diferentes manejos. Pesq Agropec Bras. 2005;40:487-94. 